$6 \int_{13}^{192} O_{5}(3)$

PPPL-2806

UC-426,427
PREPARED FOR THE U.S. DEPARTMENT OF ENERGY, UNDER CONTRACT DE-AC02-76-CHO-3073

PPPL-2806

NONSTATIONARY INTERFERENCE AND SCATTERING FROM RANDOM MEDIA

BY

RAFFI NAZIKIAN

December 1991

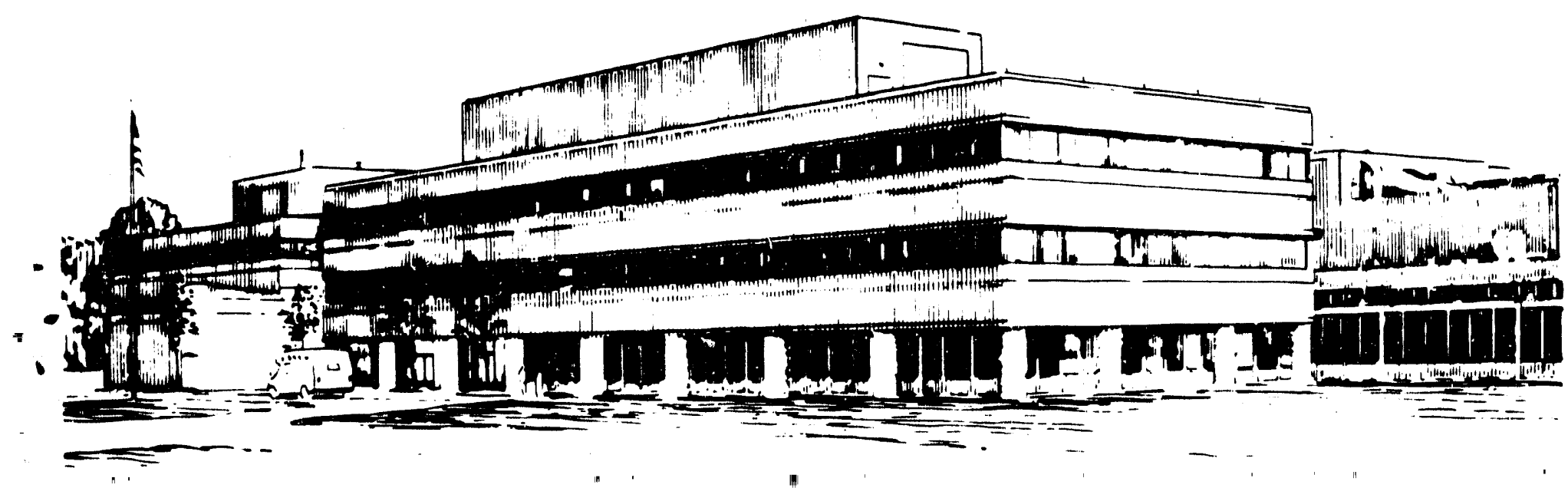




\section{NOTICE}

This report was prepared as an account of work sponsored by an agency of the United States Government. Neither the United States Government nor any agency thereof, nor any of their employees, makes any warranty, express or implied, or assumes any legal liability or responsibility for the accuracy, completeness, or usefulness of any information, apparatus, product, or process disclosed, or represerits that its use would not infringe privately owned rights. Reference herein to any specific commercial produce, process, or service by trade name, trademark, manufacturer, or otherwise, does not necessarily constitute or imply its endorsement, recommendation, or favoring by the United States Government or any agency thereof. The views and opinions of authors expressed herein do not necessarily state or reflect those of the United States Government or any agency thereof.

\section{NOTICE}

This report has been reproduced directly from the best available copy.

Available to DOE and DOE contractors from the:

Office of Scientific and Technical Information

P.O. Box 62

Oak Ridge, TN 37831 ;

Prices available from (615) 576-8401.

Available to the public from the:

National Technical Information Service

U.S. Department of Commerce 5285 Port Royal Road Springfield, Virginia 22161

$703-487-4650$ 


\title{
NONSTATIONARY INTERFERENCE AND SCATTERING FROM RANDOM MEDIA
}

\author{
Raffi Nazikian \\ Princeton Plasma Physics Laboratory \\ Princeton University, Princeton, New Jersey
}

PPPL--2806

DE92 006816

\begin{abstract}
For the small angle scattering of coherent plane waves from inhomogeneous random media, the three dimensional mean square distribution of random fluctuations may be recovered from the interferometric detection of the nonstationary modulational structure of the scattered field. Modulational properties of coherent waves scattered from random media are related to nonlocal correlations in the double sideband structure of the Fourier transform of the scattering potential. Such correlations may be expressed in terms of a suitably generalized spectral coherence function for analytic fields.
\end{abstract}


In the classical theory of partial coherence it is usually assumed that the mutual coherence function $\Gamma_{u}=\left\langle\hat{u}_{1} \hat{u}_{2}^{*}\right\rangle$ provides an appropriate representation for the second order coherence properties of optical fields where $u$ is the analytic representation of a real scalar field $u^{R}=u+u^{*}$ and $\hat{u}=u-\langle u\rangle$. One central feature of the theory is that for scalar wide sense stationary fields, the covariance of the real field $\Gamma_{u}^{R}$ is expressible in terms of the mutual coherence by the relation $\Gamma_{u}^{R}=2 \Re e \Gamma_{u}$, where the mutual correlation $\tilde{\Gamma}_{u}=\left\langle\hat{u}_{1} \hat{u}_{2}\right\rangle$ is zero by stationarity. ${ }^{1,2}$ Also, by the nature of the mutual coherence function in the optical far field, the spectral coherence of the stationary analytic field $\hat{u}$ is given by ${ }^{3}$

$$
\mu_{12}=\frac{W_{12}}{\mathcal{W}_{11}^{1 / 2} W_{22}^{1 / 2}}
$$

where $W_{12}=\left\langle U_{1} U_{2}^{*}\right\rangle$ and $U_{1}=U\left(\mathbf{k}_{S 1}, \omega_{1}\right)$ is an arbitrary coefficient of the plane wave decomposition of $\hat{u}$.

However, in the important class of problems concerning the scattering of coherent deterministic waves from random media, where the mutual correlation may be nonzero yet highly oscillatory, the mutual coherence (or its real part) is still considered the appropriate second order measure of the scattered field. ${ }^{2,4,5}$ The motivation for overlooking highly oscillatory terms such as $\left\langle\hat{u}_{1} \hat{u}_{2}\right\rangle$ in the real covariance is that it does not constitute a measurable property of the optical field, where measurability in partial coherence has traditionally been associated with the visibility of Youngs interference fringes.

In this paper we show that highly oscillatory terms such as $\left\langle\hat{u}_{1} \hat{u}_{2}\right\rangle$ may be determined using heterodyne interferometry and that such terms provide entirely new information on the three dimensional distribution of 
random media in the experimentally important limit of the small angle scattering of coherent plane waves. The mutual correlation is shown to reveal new information on the line of sight distribution of random media not conveyed by the mutual coherence function for scattering at small angles to the incident wave.

A key result in the application of coherence theory to the scattering of coherent waves from quasihomogeneous random media, is that the degree of coherence of the scattered field in the far zone is related to the mean square distribution of the scattering potential by the relation

$$
\mu\left(\mathbf{k}_{S 1}, \mathbf{k}_{S 2} ; \mathbf{k}_{0}\right) \approx \Sigma\left(\mathbf{k}_{S 1}-\mathbf{k}_{S 2}\right) / \Sigma(0), \quad\left|\mathbf{k}_{S}\right|=\left|\mathbf{k}_{0}\right|
$$

where $\Sigma$ is the Fourier transform of the mean square distribution of fluctuations and $\mathbf{k}_{S}$ is a wavevector of the scattered field. ${ }^{5}$ By Eq. (2), in the experimentally important case of the small angle scattering of coherent plane waves, the spectral coherence conveys limited information on the location or inhomogeneous distribution of the random medium in the direction of the incident wave. This is particularly so for approximately slab-like random media where $\Sigma(\mathbf{K}) \simeq \delta\left(\mathbf{K}_{\perp}\right) \Sigma_{z}\left(\mathrm{~K}_{z}\right)$ and $\mu \simeq \delta\left(\mathbf{k}_{S 1}-\mathbf{k}_{S 2}\right)$ by the Bragg scattering condition $\left|\mathbf{k}_{S}\right|=\left|\mathbf{k}_{0}\right|$. In such cases, the coherence is independent of and, hence, insensitive to the inhomogeneous distribution of the random medium in the direction of the incident wave.

However, the scattered field may be shown to convey further information on the inhomogeneous distribution of the random medium in the direction of the incident wave, independent of the degree of homogeneity of the medium normal to $\mathbf{k}_{0}$. This information is not contained within the standard measure of spectral coherence for analytic fields given by 
Eq. (1). New information on the location and three dimensional distribution of random media is shown to be contained in the nonstationary modulational structure of scattered fields which may be recovered by use of hcierodyne interferometry.

For the coherent scattering of deterministic fields, consider a secondary source generated by the interaction of monochromatic plane waves with random media. Within the Born approximation, taking $P(\mathbf{r}, t)$ as the analytic representation of the induced polarization $P^{R}$ in the medium for an incoming plane wave $u_{0}=\exp \left(i \mathrm{k}_{0} z-i \omega_{0} t\right)$, then

$$
P(\mathbf{r}, t)=\eta\left(\mathbf{r}, t ; \omega_{0}\right) u_{0}(\mathbf{r}, t)
$$

where $\eta$ is the real susceptibility assuming $\omega_{0}$ is sufficiently removed from any resonances and assuming the bandwidth $\Omega_{M}$ of fluctuations is very narrow $\left(\Omega_{M} \ll \omega_{0}\right){ }^{6}$ The covariance of the real polarization is then given by

$$
\Gamma_{P}^{R}=2 \Re e\left[\Gamma_{P}+\tilde{\Gamma}_{P}\right]
$$

where $\Gamma_{P}^{R}=\left\langle\hat{P}_{1}^{R} \hat{P}_{2}^{R}\right\rangle, \Gamma_{P}=\left\langle\hat{P}_{1} \hat{P}_{2}^{*}\right\rangle$ is the mutual oherence and $\tilde{\Gamma}_{P}=$ $\left\langle\hat{P}_{1} \hat{P}_{2}\right\rangle$ is the highly oscillatory mutual correlation of the source. Assuming the real susceptibility is wide sense stationary with covariance $C_{\eta}=\left\langle\hat{\eta}_{1} \hat{\eta}_{2}\right\rangle$, then the nonstationarity of the mutual correlation is evident from the deterministic nature of $u_{0}$,

$$
\tilde{\Gamma}_{P}=u_{0}\left(\mathbf{r}_{1}, t_{1}\right) u_{0}\left(\mathbf{r}_{2}, t_{2}\right) C_{\eta}
$$

We then have for the covariance of the scattered field,

$$
\Gamma_{u}^{R}=2 \Re e \iint_{V_{P}} d r^{\prime} d r^{\prime \prime}\left[h_{\mathrm{r}_{1}, \mathbf{r}^{\prime}} h_{\mathbf{r}_{2}, \mathrm{r}^{\prime \prime}}^{*} \Gamma_{P}+h_{\mathrm{r}_{1}, \mathbf{r}^{\prime}} h_{\mathrm{r}_{2}, \mathrm{r}^{\prime}} \tilde{\Gamma}_{P}\right]
$$


where $h$ is the appropriate Greens function for the free space propagation of the analytic field $u$ with source function $P$ satisfying the inhomogeneous Helmholtz wave equation. In Eq. (6), $\hat{\eta}$ is assumed not to vary over the propagation time of the field through the medium to the observer plane. $\tilde{\Gamma}_{P}$ is by Eq. (6) the source term for $\tilde{\Gamma}_{u}$ in the covariance of the scattered field. Unlike the mutual coherence, the highly oscillatory nature of $\tilde{\Gamma}_{u}$ (for $t^{\prime}-t=$ const.) makes it inaccessible to direct measurement via a Youngs interference (or Michelson interferometer) experiment which recovers terms proportional to $2 \Re e\left[u_{1} u_{2}^{*}\right]$, related to the measurability of interference fringes via the relation [ c.f. Fig. 1(a)]

$$
I(\mathbf{r}, t) \propto\left|u_{1}\right|^{2}+\left|u_{2}\right|^{2}+2 \Re e\left[u_{1} u_{2}^{*}\right],
$$

where $u_{i}=u\left(\mathbf{r}_{i}, t-\tau_{i}\right)$. Fringes result from relative path delays $\tau_{i}$ in the propagation of the field to the point of measurement. ${ }^{1,4}$ Terms such as $u_{1} u_{2}$ are typically ignored in the classical representation of the instantaneous intensity by the physical requirement that square law detectors integrate for a time $\tau_{\text {int }} \gg 2 \pi / \omega_{0}$, although such detectability criteria do not naturally arise out of the classical theory.

We instead address the question of measurability by considering the heterodyne detection of the scattered field as shown in Fig. 1(b) where the local oscillator $u^{\prime}=\exp \left[i\left(\mathrm{k}_{0}^{\prime} z-\omega_{0}^{\prime} t+\phi_{0}\right)\right]$ is derived from the same coherent source as $u_{0}$ but doppler shifted down in frequency to $\omega_{0}-\Omega_{0}$ via some mechanical or acousto-optic technique such that $\Omega_{M} \ll \Omega_{0} \ll \omega_{0}$. The measurable oscillatory component of the interference is then given by $v^{R}=2 \Re e\left[u u^{\prime *}\right]$. If we require $\Omega_{0} \gg \Omega_{M}$ to avoid ambiguity in the sign of measured frequency shifts in the scattered field, then $v=u u^{\prime *}$ is the 
appropriate analytic representation of the real interference. In terms of the analytic signal $v$, the covariance of $v^{R}$ is given by

$$
\begin{aligned}
& \Gamma_{v}^{R}=2 \Re e\left[\Gamma_{v}+\tilde{\Gamma}_{v}\right], \\
& \text { where } \Gamma_{v}=u_{1}^{\prime *} u_{2}^{\prime} \Gamma_{u} \\
& \text { and } \quad \tilde{\Gamma}_{v}=u_{1}^{\prime *} u_{2}^{\prime *} \tilde{\Gamma}_{u}
\end{aligned}
$$

By Eq. (8), the mutual correlation of $v^{R}$ is nonzero and oscillates with the much lower and measurable frequency $2 \Omega_{0}$ instead of $2 \omega_{0}$ for the covariance of the scattered field. For simplicity we have assumed $u_{0}$ is deterministic in order to demonstrate the correspondence between the covariance of the scattered field and that of the measurable interference. However if $u_{0}$ is not assumed deterministic such that $\left\langle u_{0}\right\rangle=0$ and $\tilde{\Gamma}_{u}=$ 0 , the covariance of $v^{R}$ remains nonstationary provided $u_{0} u^{\prime *}$ remains deterministic as in the heterodyne detection of highly coherent optical or microwave sources. As $C_{\eta}$ is wide sense stationary, $\Gamma_{v}^{R}$ is periodic in $t=\left(t_{1}+t_{2}\right) / 2$ with period $\pi / \Omega_{0}$. The detectable interference $v^{R}$ is representative of the class of harmonically modulated random fields. General properties of one dimensional random modulational fields may be found in the literature on nonstationary processes. ${ }^{7}$

The appearance of nonstationarity in the scattered field results from noniocal correlations in the Fourier transform of the source. Unlike thermal sources (which may be considered a linear superposition of uncorrelated harmonic oscillators), modulated fields are characterized by correlated sidebands symmetrically displaced about the carrier frequency $\left(\mathbf{k}_{0}, \omega_{0}\right)$. The statistical correlation between equispaced sidebands about the carrier frequency is conveyed by the Fourier transform of the non- 
stationary mutual correlation. The degree of correlation between symmetrically displaced sidebands in the forward scattered field provides new information on the three dimensional distribution of randomly modulated coherent sources i.e., scattering potentials.

By Eq. (3), the polarization represents the spatial and temporal modulations impressed on $u_{0}$ by irregularities in the medium. For example, an arbitrary real wave of frequency $(K, \Omega)$ in any one realization of the random medium modulates the incident wave generating symmetrically displaced sidebands at frequencies $\left(\mathbf{k}_{0} \pm \mathbf{K}, \omega_{0} \pm \Omega\right)$ in the Fourier transform of the polarization. Taking the Fourier transform of $\tilde{\Gamma}_{P}$ and making use of the relation $\eta_{\mathbf{K}, \Omega}=\eta_{-\mathbf{K},-\Omega}^{*}$ for the Fourier transform of $\hat{\eta}$ leads to the expected correlation between opposite sidebands of the source,

$$
\left\langle\mathcal{P}_{\mathbf{k}_{1}, \omega_{1}} \mathcal{P}_{\mathbf{k}_{2}, \omega_{2}}\right\rangle \propto\left\langle\eta_{\mathbf{k}_{1}-\mathbf{k}_{0}, \omega_{1}-\omega_{0}} \eta_{\mathbf{k}_{0}-\mathbf{k}_{2}, \omega_{1}-\omega_{0}}^{*}\right\rangle\left(\omega_{1}+\omega_{2}-2 \omega_{0}\right) .
$$

We may interpret the origin of the nonstationarity in the source to the correlation between symmetrically displaced spectral lines in the polarization. This correlation may in turn be considered characteristic of randomly modulated coherent fields, analogous to the double sideband structure of amplitude and frequency modulated radio waves. If on the other hand the incident wave is assumed to have no deterministic component, then the correlation between opposite sidebands disappears as in the recently discussed case of the scattering of random fields $\mu \mathrm{Jm}$ random media. ${ }^{6,8}$ By further considering the symmetry properties of scattered fields from the perspective of the Fourier diffraction projection theorem, ${ }^{9,10}$ we show that the correlation between opposite sidebands in the scattered field, as measured on an arbitrary plane in front of the 
source, relays information on the distribution of the random medium in the direction of the incident wave.

For heterodyne interferometry, we are specifically interested in the planar measurement of the scattered field at a fixed (but arbitrary) $z_{0}$. Such a geometry is motivated by the experimental constraint of detecting the scattered field on a planar or linear array of heterodyne receivers. ${ }^{11}$ The planar Fourier transform $U_{\mathbf{K}_{\perp, \omega}}$ of the scattered field $\hat{u}\left(\mathbf{r}_{\perp}, t ; z_{0}\right)$ is related to the spectrum of the polarization by the expression

$$
U_{\mathbf{K}_{\perp, \omega}} \propto \frac{\pi \mathrm{i}}{\mathbf{k}_{\|}} e^{i \mathbf{k}_{\|} z_{0}} \mathcal{P}_{\mathbf{K}_{\perp, \mathbf{k}_{\|}, \omega}}
$$

where $\mathbf{k}_{\|}=\sqrt{\mathbf{k}_{0}^{2}-\mathrm{K}_{\perp}^{2}}$ is positive, consistent with forward scattering. ${ }^{12}$ Inserting $U_{\mathbf{K}_{\perp, \omega}}$ into the expression for $V_{\mathbf{K}_{\perp}, \Omega}$ (the Fourier transform on the plane $z=z_{0}$ of the analytic signal $\hat{v}$ of the measurable interference $\left.\hat{v}^{R}\right)$ we obtain

$$
V_{\mathbf{K}_{\perp}, \Omega_{0}+\Omega} \propto e^{i \mathrm{~K}_{\|} z_{0}} \eta_{\mathbf{K}_{\perp}, \mathbf{K}_{\|}, \Omega}
$$

where $K_{\|} \simeq-K_{\perp}^{2} / 2 k_{0}$, which is valid within the parabolic approximation for the small angle scattering of high frequency waves. Apart from various phase factors, Eq. (11) is a restatement of the Bragg condition for the elastic scattering of coherent waves where $\mathbf{k}_{S}=\mathbf{k}_{0}+\mathbf{K}$ subject to the constraint $\left|\mathbf{k}_{S}\right|=\left|\mathbf{k}_{0}\right|$. For sufficiently small scattering angles, $\mathbf{k}_{S} \simeq\left(\mathbf{K}_{\perp}, \mathbf{k}_{0}+\mathbf{k}_{\|}\right)$. The locus of all points in $\mathbf{k}$-space satisfying the Bragg condition is illustrated in Fig. 2 by the parabolic slice through the Fourier transform of the medium. The graphical representation of the Bragg condition makes clear the connection between dlastic scattering in optics and the Fourier projection theorem in tomography. ${ }^{9,10}$ Impor- 
tantly, note that only the half space $K_{z}<0$ contributes to the spectrum of the scattered field. An interesting consequence of elastic scattering is that the spectrum of the scattered field need not retain the same symmetry between opposite sidebands about $\left(\mathbf{k}_{0}, \omega_{0}\right)$ as does the spectrum of the polarization in Eq. (9). Specifically, although the polarization retains complete information on the spectral distribution of the random medium, only a small asymmetric component of the source spectrum is radiated to the observer plane. We may, however, make use of this asymmetry in the scattered field to further investigate the inhomogeneous distribution of the source in the direction of the incident wave. As in Eq. for the polarization, taking the correlation between opposite sidebands $V_{ \pm}=V_{ \pm \mathbf{K}_{\perp}, \Omega_{0} \pm \Omega}$ on the detector plane gives

$$
\left\langle V_{+} V_{-}\right\rangle \propto e^{2 i \mathrm{~K}_{\| z_{0}}}\left\langle\eta_{+} \eta_{-}^{*}\right\rangle
$$

where $\eta_{ \pm}=\eta_{\mathbf{K}_{\perp}, \pm \mathrm{K}_{\|}, \Omega}$ and $\Omega_{0} \pm \Omega_{M}>0$. Importantly, the asymmetric distribution of the scattered field in $K_{z}$ (shown in Fig. 2) allows the coherence between the two Fourier components $\eta_{\mathbf{K}_{\perp, \pm K_{\|}, \Omega}}$ displaced by $\mathrm{K}_{z}=2 \mathrm{~K}_{\|}$to be recovered from the correlation between the two Fourier components $V_{ \pm \mathbf{K}_{\perp}, \Omega_{0} \pm \Omega}$ of the interference. This result seems intuitively surprising as those components with $\mathrm{K}_{z}>0$ do not directly contribute to the scattered field. By Eq. (12), the Fourier transform of the mutual correlation appears to convey new information on che inhomogeneous distribution of the random medium in the direction of the incident wave, independent of the spectral coherence (homogeneity) of the random medium normal to $\mathbf{k}_{0}$. This result appears at odds with Eq. (2) derived from the standard measure of spectral coherence for the analytic field. ${ }^{5}$ 
Formally, we define the generalized spectral coherence of the analytic signal $\hat{v}$ by the expression

$$
\mu_{12}^{v}=\frac{\mathcal{W}_{12}^{v}}{\left[\mathcal{W}_{11}^{v}\right]^{1 / 2}\left[\mathcal{W}_{22}^{v}\right]^{1 / 2}}
$$

where $\mathcal{W}_{12}^{v}=\mathcal{W}_{12}+\tilde{W}_{12}, \mathcal{W}_{12}=\left\langle V_{1} V_{2}^{*}\right\rangle$ is the cross spectral density and $\tilde{W}_{12}=\left\langle V_{1} V_{2}\right\rangle$ in the cross spectral correlation. An illustration of the nonlocal nature of the cross spectral correlation in the Fourier transform plane of the signal is shown in Fig. 3 . In terms of $\mathcal{W}$ and $\tilde{W}$, the generalized spectral coherence becomes

$$
\mu_{12}^{v}=\frac{\mathcal{W}_{12}+\tilde{\mathcal{W}}_{12}}{\mathcal{W}_{11}^{1 / 2} \mathcal{W}_{22}^{1 / 2}}
$$

where $\tilde{\mathcal{W}}_{11}=0$ by the nonstationarity of $\tilde{\Gamma}_{v}$. The first term in Eq. (14) is the usual expression for the spectral coherence of stationary analytic fields whereas the second term is the new component of the spectral coherence associated with the nonstationary structure of modulated fields.

We now introduce the widely used model assumption of quasihomogeneity for the random medium, $C_{\eta}=I(\overline{\mathbf{r}}) \rho\left(\mathbf{r}^{\prime}, t^{\prime}\right)$, where $\rho$ is the real symmetric correlation function and $I(\overline{\mathbf{r}})=\left\langle|\hat{\eta}|^{2}\right\rangle$ is the inhomogeneous distribution of the random medium where $\overline{\mathbf{r}}=1 / 2\left(\mathbf{r}_{1}+\mathbf{r}_{2}\right), \mathbf{r}^{\prime}=\mathbf{r}_{1}-\mathbf{r}_{2}$ and $t^{\prime}=t_{1}-t_{2}$. The cross spectral density $C_{\eta}=\left\langle\eta_{K_{1} \Omega_{1}} \eta_{K_{2}, \Omega_{2}}^{*}\right\rangle$ of the susceptibility is given by

$$
c_{\eta}=\Sigma\left(\mathbf{K}_{1}-\mathbf{K}_{2}\right) \mathrm{S}(\overline{\mathbf{K}}, \bar{\Omega}) \delta\left(\Omega_{1}-\Omega_{2}\right)
$$

where $\Sigma$ (the Fourier transform of $I$ ) represents the shift invariant spectral coherence function and $S$ is the real symmetric Fourier transform of the two point correlation $\rho$ where $(\overline{\mathbf{K}}, \bar{\Omega})=\left[1 / 2\left(\mathbf{K}_{1}+\mathbf{K}_{2}\right), 1 / 2\left(\Omega_{1}+\Omega_{2}\right)\right]$. 
An important consequence of quasi-homogeneity is that $S(\mathrm{~K}, \Omega)$ is uniform about any $\mathbf{K}$ within a range $\Delta \mathbf{K}$ determined by the spectral width of the coherence function $\Sigma$ (c.f. Fig. 2). ${ }^{5}$ Within the range where $|\Sigma(\Delta \mathbf{K}) / \Sigma(0)| \sim 1$, Eq. (14) becomes

$$
\mu_{12}^{v} \approx \mu_{-} \delta\left(\Omega_{-}\right)+\mu_{+} \delta\left(\Omega_{+}\right),
$$

where

$$
\begin{aligned}
& \mu_{ \pm}=\exp \left(i \mathbf{K}_{ \pm} \cdot \hat{\mathbf{k}}_{0} z_{0}\right) \Sigma\left(\mathbf{K}_{ \pm}\right) / \Sigma(0), \\
& \Omega_{ \pm}=\left(\Omega_{1}-\Omega_{0}\right) \pm\left(\Omega_{2}-\Omega_{0}\right), \\
& \mathbf{K}_{ \pm}=\left(\mathbf{K}_{\perp 1} \pm \mathbf{K}_{\perp 2}, \mathbf{K}_{\| 1} \pm \mathbf{K}_{\| 2}\right) .
\end{aligned}
$$

The relative phase $\phi_{0}$ between $u_{0}$ and $u^{\prime}$ is nominally set to $\pi / 2$ at $t=0$ and $\hat{\mathbf{k}}_{0}=\mathbf{k}_{0} /\left|\mathbf{k}_{0}\right|$. In Eq. (16), $\mu_{-}$may be derived from the standard measure of spectral coherence [ c.f. Eqs. (1)\&(2)] whereas $\mu_{+}$resembles the normalized spectral correlation derived from the time independent complex phase of the scattered field. ${ }^{13}$ Note that $\mu_{+}$may sample a much wider coherence range in $\mathrm{K}_{z}$ than $\mu_{-}$by taking nonlocal correlations between opposite sidebands $V_{ \pm \mathbf{K}_{\perp}, \Omega_{0} \pm \Omega}$ on the plane of measurement. Given $\mathrm{K}_{0}$ is the maximum resolved wavenumber on the detector plane then the sampled coherence range in $K_{z}$ is $\Delta K_{z}=K_{0}^{2} / k_{0}$, as illustrated in Fig. 2. Considering only the nonstationary component of the covariance and introducing two new independent variables $K_{\perp}^{\prime}=K_{\perp 1}+K_{\perp 2}$ and $K_{z}^{\prime}=-\left|K_{\perp 1}-K_{\perp 2}\right|^{2} / 4 k_{0}$ gives

$$
\mu_{+} \approx e^{i K_{z}^{\prime} z_{0}} \Sigma\left(\mathbf{K}_{\perp}^{\prime}, K_{z}^{\prime}\right) / \Sigma(0)
$$

which is valid within the parabolic and quasi-homogeneity approximations $\Delta \mathrm{K}_{\perp} / \mathrm{k}_{0} \ll \mathrm{K}_{0} / \mathrm{k}_{0} \ll 1$, where $\Delta \mathrm{K}_{\perp}$ is the spectral width of $\Sigma$ normal to $\mathbf{k}_{0}$. Note that the form of Eq. (17) is identical to the three 
dimensional Fourier transform of $I(\mathbf{r})$ relative to the location of the detector plane. Hence, taking the inverse Fourier transform of $\mu_{+}$over the measurable spectral range gives

$$
I_{B}(\mathbf{r}) / I_{0} \approx 2 \Re e \int_{-\mathrm{K}_{0}}^{\mathrm{K}_{0}} \frac{d \mathbf{K}_{\perp}^{\prime}}{(2 \pi)^{2}} \int_{-\mathrm{K}_{0}^{2} / k_{0}}^{0} \frac{d \mathrm{~K}_{z}^{\prime}}{2 \pi} e^{i \mathbf{X}^{\prime} \cdot\left(\mathbf{r}-\mathbf{r}_{0}\right)} \mu_{+}\left(\mathbf{K}^{\prime}\right)
$$

where $I_{0}=\Sigma(0), \mathbf{r}_{0}=\hat{\mathbf{k}}_{0} z_{0}$ and $I_{B}$ is given by $I(\mathbf{r})$ band limited to $\Delta \mathrm{K}_{z}=\mathrm{K}_{0}^{2} / \mathrm{k}_{0}$. Both the absolute location and three dimensional distribution of random media may then be recovered from the measurement of nonstationary correlations in the forward scattered field. Intuitively, the correlation between symmetrically displaced diffraction orders provides a stereoscopic view of the three dimensional inhcmogeneous distribution of random media not contained within the stationary component of the covariance. In an analogy with tomography, information contained in the nonstationary correlation between opposite sidebands of the forward scattered field is qualitatively similar to information which may be recovered from the cross correlation between two geometric projections of the random medium separated by a relative angle $\theta=K_{0} / k_{0}$.

Existing experimental techniques based on the coherent detection of optical, microwave or acoustic waves scattered from rardom media may readily be adapted to investigate nonstationary properties of the measurcu signal. The application of this measure to existing multichannel imaging and scattering experiments will be discussed elsewhere.

\section{Acknowledgments}

I would like to thank Dr. M. Yamada for many helpful discussions. This work was supported by the U.S. Department of Energy contract No. DEAC02-76-CHO-3073. 


\section{References}

[1] L. Mandel and E. Wolf, Rev. Mod. Phys., 37, 231 (1965).

[2] S.M. Rytov, Yu.A. Kravtsov, and V.I. Tatarskii, Principles of Statistical Radiophysics translated by A.P. Repyev (Springer-Verlag 1987). See Ch. 2.6, Vol. 2 for a discussion of nonstationary interference and Ch. 1.6, Vol. 4 on the optical coherence function for scattered fields.

[3] W.H. Carter and E. Wolf, Optica Acta, 28, 227 (1981).

[4] M.J. Beran and G.B. Parrent Jr., Theory of Partial Coherence (PrenticeHall International series 1964), see Ch. 2.6 for a discussion of measurability and Ch. 6 on scattering from random media.

[5] W.H. Carter and E. Wolf, Opt. Commun., 67, 85 (1988).

[6] E. Wolf and J.T. Foley, Phys. Rev. A, 40, 579 (1989).

[7] J.S. Bendat and A.G. Piersol, Random Data (John Wiley \& Sons 1986) see Ch. 12 on nonstationary data analysis.

[8] L. Mandel and E. Wolf, Opt. Commun., 8, 95 (1973).

[9] E. Wolf, Opt. Commun., 1, 153 (1969).

[10] J. Howard, W.A. Peebles, D.L. Brower, S.K. Kim, and N.C. Luhmann, Jr., Proceeding of the $8^{\text {th }}$ Topical Conference on High Temperature Plasma Diagnostics. 6-10 May, 1990. Rev. Sci. Instrum., 61, 2829 (1990).

[11] R. Nazikian and L. E. Sharp, Rev. Sci. Instrum., 58, 2086 (1987). 
[12] W. C. Chew, Waves and Fields in Inhomogeneous Media, (Van Nostrand Reinhold, New York 1990). See Ch. 2.2 on the spectral representation of sources and fields.

[13] R. Nazikian, Proceeding of the $8^{\text {th }}$ Topical Conference on High Temperature Plasma Diagnostics. 6-10 May, 1990. Rev. Sci. Instrum., 61, 3004 (1990). 


\section{Figure Captions}

Fig. 1. The visibility of Youngs interference fringes [ shown in 1(a)] conveys information on the mutual coherence of random optical fields. In 1 (b), both the mutual coherence and highly oscillatory mutual correlation may be recovered from the interferometric detection of the scattered field.

Fig. 2. The correlation between symmetrically displaced sidebands $V_{ \pm \mathbf{K}_{\perp}, \Omega_{0} \pm \Omega}$ on the detector plane is a measure of the coherence between the two Fourier components $\eta_{\mathbf{K}_{\perp}, \pm K_{\|}, \Omega}$ in the random medium. Shaded regions indicate the range of spectral coherence about any wavenumber.

Fig. 3. The cross spectral density $\mathcal{W}_{12}$ and the nonlocal cross spectral correlation $\overline{\mathcal{W}}_{12}$ are shown in terms of correlations between appropriate components of the planar Fourier transform of the measurable interference. 


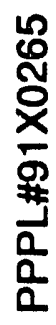
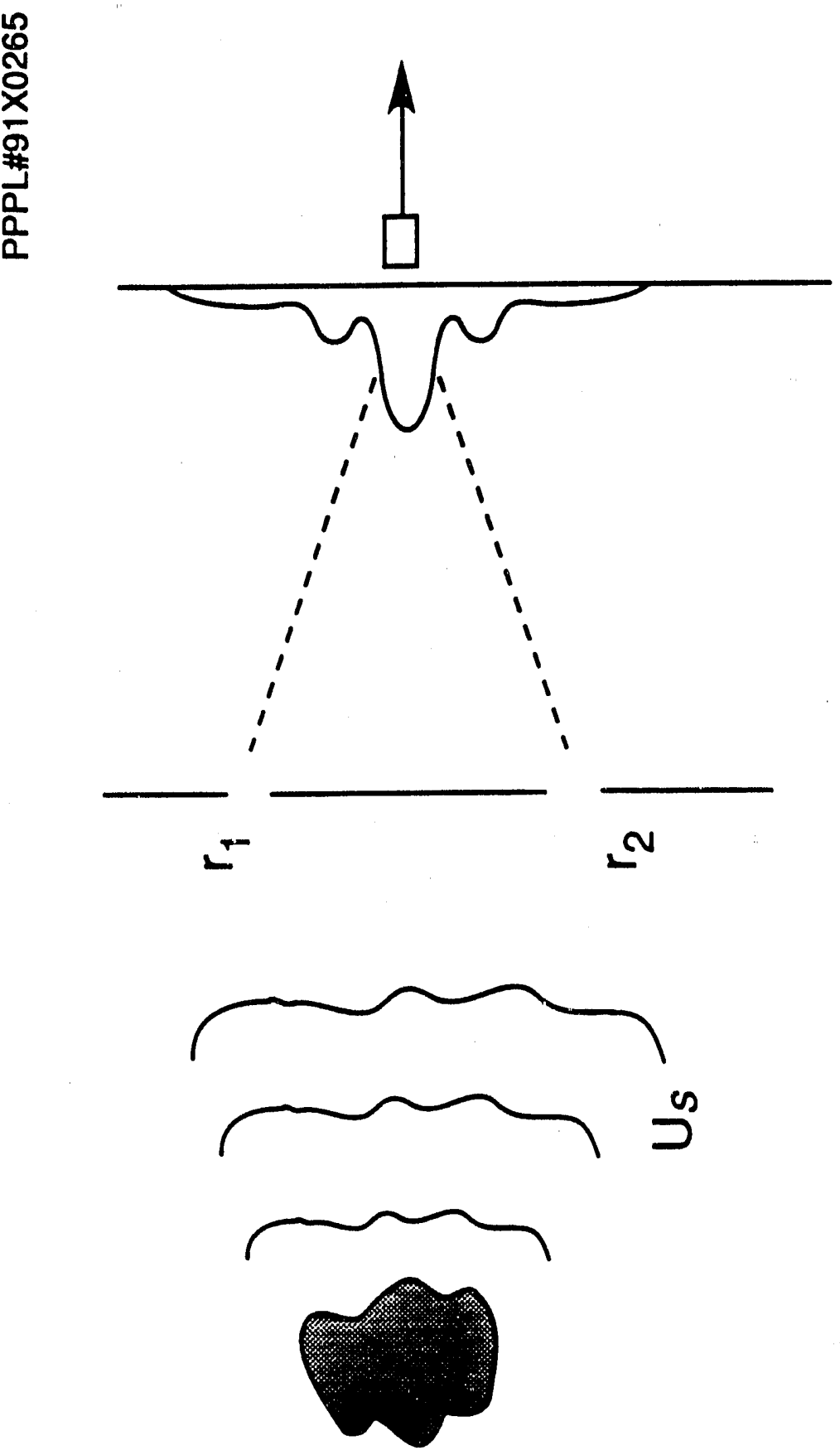

త్

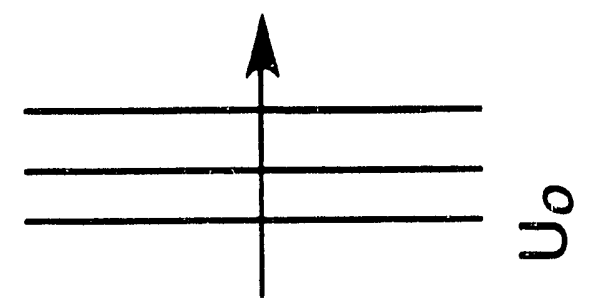

오
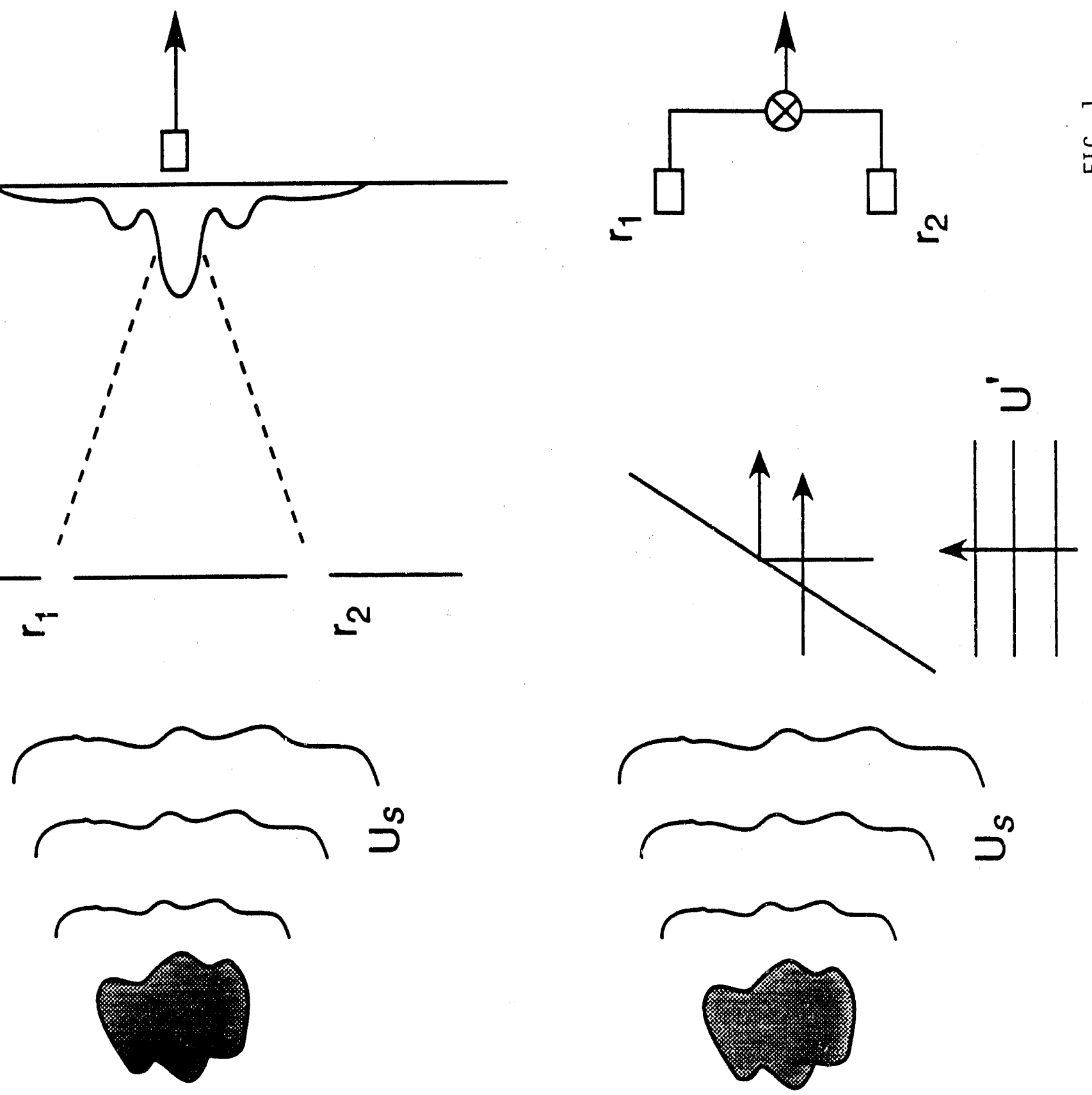

a

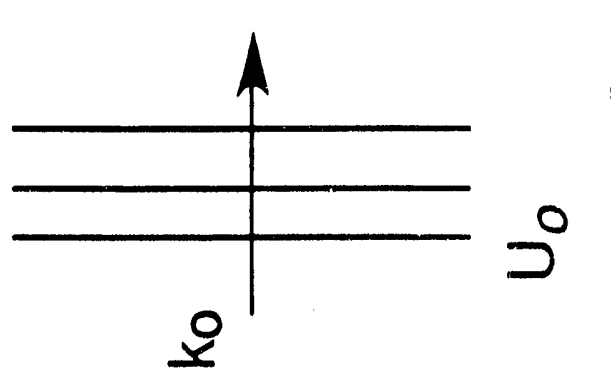


PPPL\#90X0279

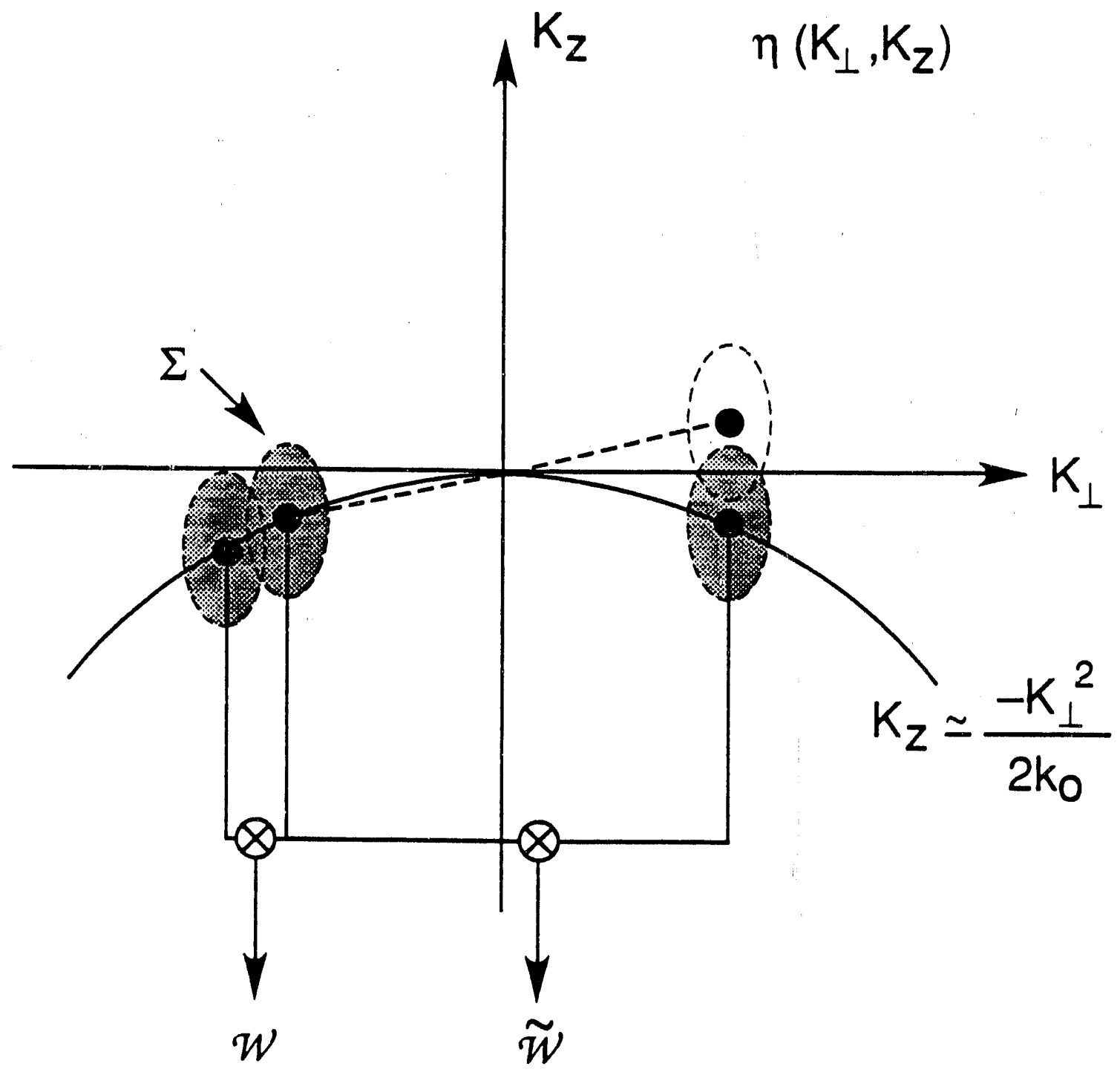

FIG. 2 
0
0
0
0
0
0
$\vdots$
$\frac{1}{0}$
$\frac{1}{2}$

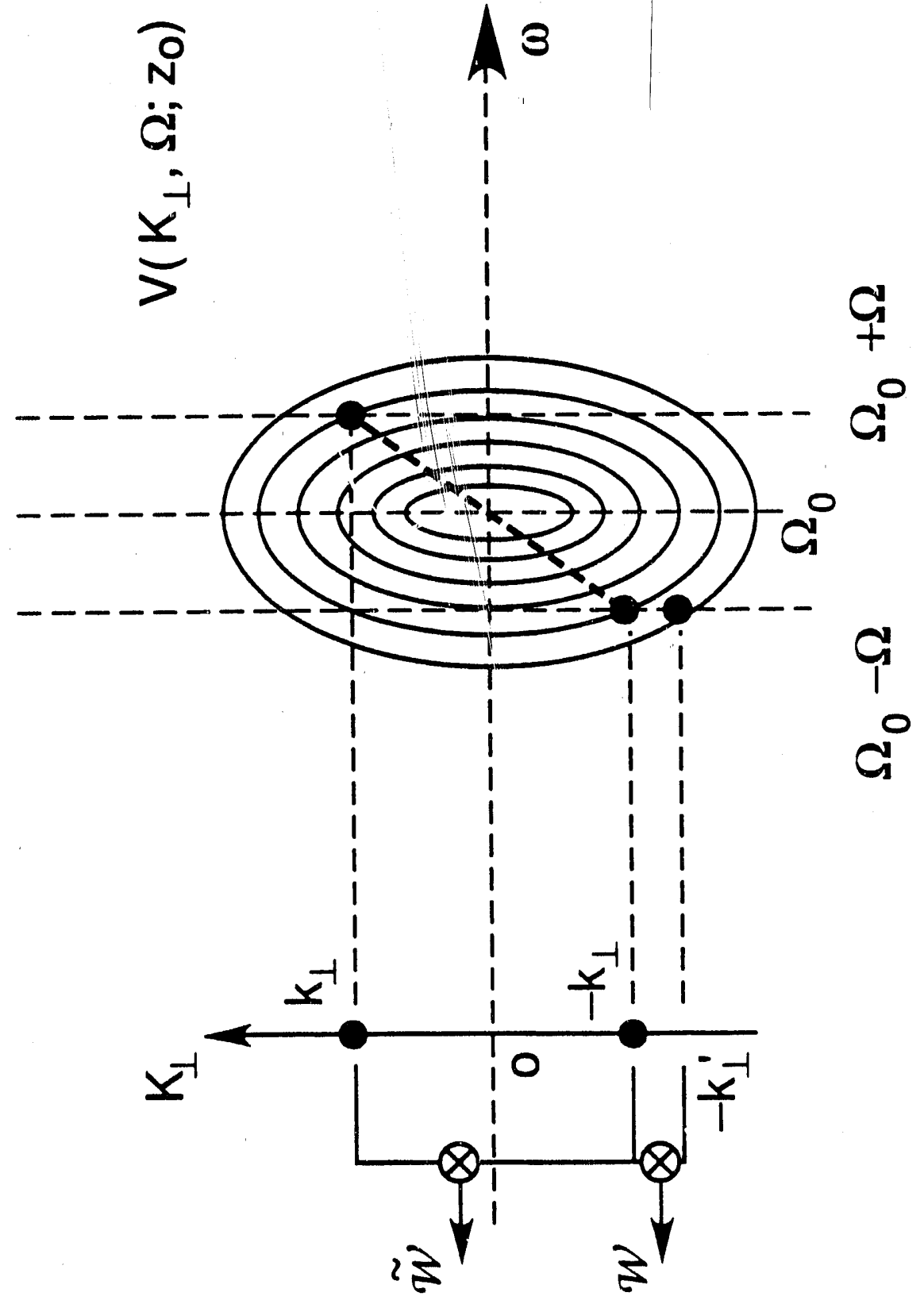

$m$
$\dot{H}$ 
Dr. F. Paoloni, Univ, of Wollongong, AUSTRALIA

Prof. M.H. Brennan, Univ. of Sydney, AUSTRALIA

Plasma Research Leb., Australien Nat. Univ., AUSTRALIA

Prof. I.R. Jones, Flindors Univ, AUSTRALIA

Prof. F. Cap, Inst. for Theoretical Physics, AUSTRIA

Prof. M. Heindter, Institut for Theoretische Physik, AUSTRIA

Prof. M. Goossens, Astronomisch InstituUt, BELGIUM

Ecole Royalo Milibire, Lab. do Phy. Plasmas, BELGIUM

Commission-European, DG. XII-Fusion Prog., BELGIUM

Prof. A. Bouciquo, Rijkeuniversibit Gent, BELGIUM

Dr. P.H. Sakanaka, Instituto Fisica, BRUZIL

Inatituto Neciond De Peequicas Espacisie-INPE, BRUZIL

Documents Office, Abmic Enorgy of Cenada Lid., CANADA

Dr. M.P. Bectyrneki, MPB Technologies, Inc., CANADA

Dr. H.M. Skarsgard, Univ. of Saskakchewan, CANA.DA

Prof. J. Teichmann, Univ. of Montreal, CANADA

Prof. S.R. Sroenivasen, Univ. of Calgary, CANADA

Prof. T.W. Johnston, INRS-Energie, CANADA

Dr. R. Bolton, Centre canedien de husion magnotique, CANADA

Dr. C.R. James,. Univ. of Aberta, CANADA

Dr. P. Luktc, Komenskeho Universzita, CZECHOSLOVAKIA

The Librarien, Cutham Leboratory, ENGLAND

Library, R61, Ruthertond Apploion Laboratory, ENGLAND

Mrs. S.A. Mutchinson, JET Library, ENGLAND

Dr. S.C. Shama, Univ. of South Pacific, FIJI ISLANDS

P. Mahonen, Univ. of Helsinki, FINLAND

Prof. M.N. Bussac, Ecole Polytochnique, FRANCE

C. Mouttet, Lab. de Physique des Milioux lonisés, FRANCE

J. Radot, CEN/CADARACHE - Bat 506, FRANCE

Prot. E. Economou, Univ. of Crete, GREECE

Ms. C. Pinni, Uniw. of loamina, GREECE

Dr. T. Mud, Acactarey Brbliographic Ser., HONG KONG

Proprint Librery, Hungarien Acodomy of Sa., HUNGARY

Dr. B. DasGupta, Saha Inst. of Nuciear Ptysics, INDIA

Dr. P. Kaw, Inst. for Plasma Research, INDIA

Dr. P. Rosenau, lereet Inst. of Technology, ISPAEL

Librarian, Intermational Center for Theo Physics, ITALY

Miss C. De Palo, Associazione EURATOM-ENEA, ITALY

Dr. G. Grosso, Istituto di Fisica del Plasma. ITALY

Prof. G. Rostangni, Istituto Gas lonizzati Del Cnr, ITALY

Dr. H. Yamato, Toshiba Ros a Dovel Centor, JAPAN
Prof. I. Kawakemi, Hiroshima Univ., JAPAN

Prot. K. Nishikawa, Hiroshima Univ., JAPAN

Director, Jepen Atomic Energy Research Inst, JAPAN

Prof. S. Itoh, Kyushu Univ., JAPAN

Research Into. Ctr., National Instit. for Fusion Science, JAPAN

Prof. S. Tanaka, Kyoto Univ., JAPAN

Libray, Kyob Univ., JAPAN

Prof. N. Inowe, Univ. of Tokyo, JAPAN

Secretary, Pleama Section, Electrolschnical Lab., JAPAN

S. Mori, Tectnical Advicor, LAEPI, JAPAN

Dr. O. Miturai, Kumamoso Inst. of Techinology, JAPAN

J. Hyeon-Sook, Korea Alomic Enorgy Rosearch Inst., KOREA

D.I. Choi, The Korea Adv. Inst. of Sai. \& Tech., KOREA

Prol. B.S. Liloy, Univ. of Weikato, NEW ZEALAND

Inst of Physica, Chinese Acad Sci PEOPLE'S REP. OF CHINA

Libray, Inst. of Plasma Physics, PEOPLE'S REP. OF CHINA

Tsinghua Univ. Library, REOP'E'S REPUBLIC OF CHINA

2. Li, S.W. Inte Phyeics, PEOPLE'S REPUBLIC OF CHINA

Prof. J.A.C. Cabred, Instiuto Superior Tecrico, PORTUGAL

Dr. O. Potrus, AL I CUZA Univ., ROMANIA

Dr. J. de Villiers, Fusion Studies, AEC, S. AFRICA

Prot. M.A. Hellberg, Univ. of Natal, S. AFRICA

Prol. D.E. Kim, Pohang Inst. of Sa. A Tech., SO. KOREA

Prot. C.I.E.M.A.T, Fusion Division Library, SPAIN

Dr. L Stonflo, Univ. of UMEA, SWEDEN

Librey, Royad Inat. of Technology, SWEDEN

Prot. H. Withotmeon, Chalmers Univ. of Tech., SWEDEN

Contre Phys. Des Plasmas, Ecole Polytech, SWITZERLAND

Bibliotheok, Inst. Voor Plasma-Fysica, THE NETHERLANDS

Asst Prot. Dr. S. Ceikir, Midde East Tech. Univ., TURKEY

Dr. V.A. Gukhikh, Sci. Res. Inst. Electrophys.I Apparatus, USSR

Dr. D.D. Ayutov, Siberian Branch of Academy of Sa., USSR

Dr. G.A. Eliseov, I.V. Kurchatov Inst, USSA

Librarian, The Ukr.SSR Academy of Sciences, USSR

Dr. LM. Kovrizhmykh, Inst. of Genoral Physics, USSR

Kerntorschungsanlege GmbH, Zentralbibliothek, W. GERMANY

Bibliothok, Inst. For Plasmatorschung, W. GERMANY

Prof. K. Schinder, Ruhr-Universitat Bochum, W. GERMANY

Dr. F. Wagner, (ASDEX), Max-Planck-Institut, W. GERMANY

Librarian, Max-Plenck-Institut, W. GERMANY

Prof. R.K. Janev, Inst. of Physics, YUGOSLAVIA 

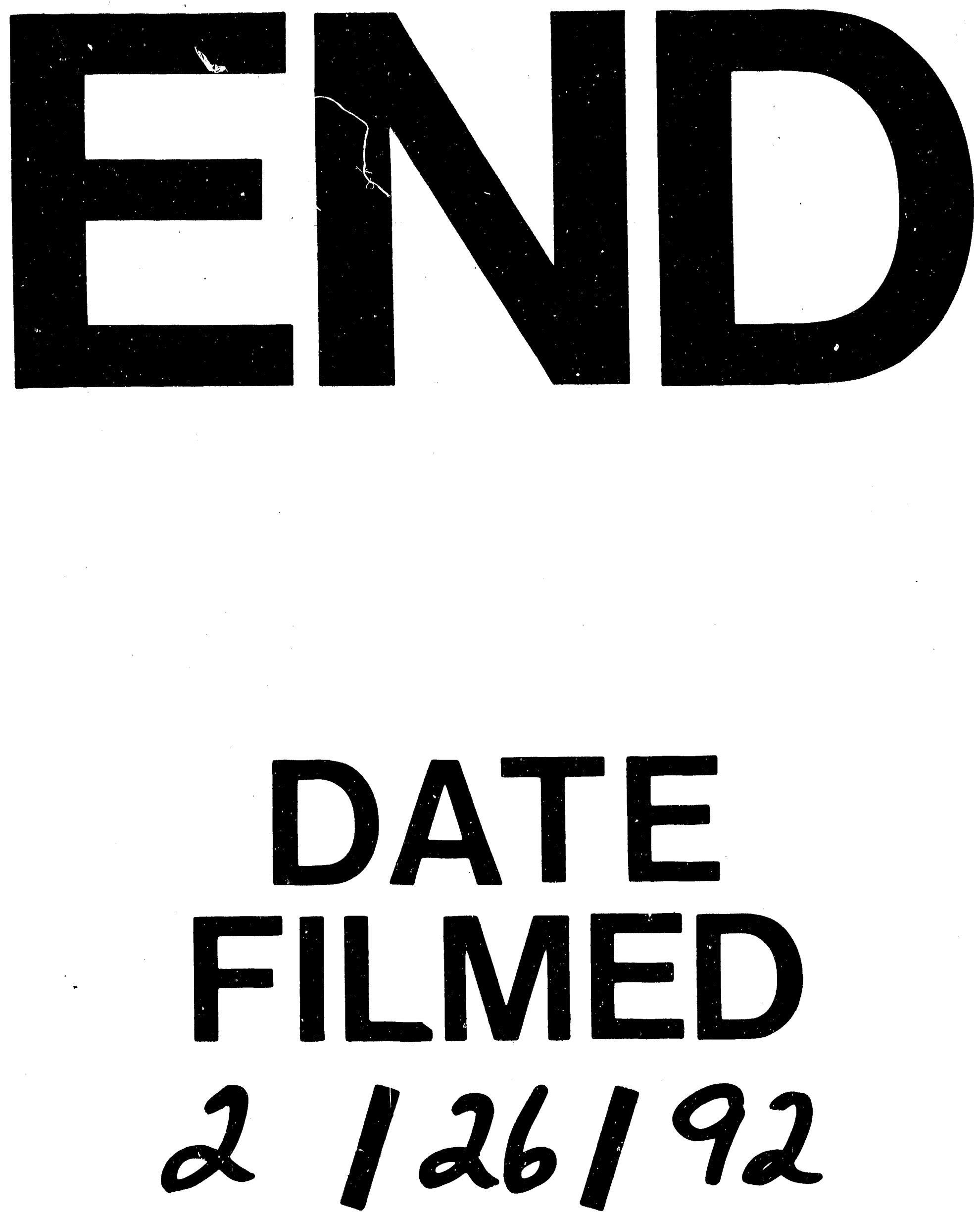
